\title{
Focus Area on Geodetic Space Weather Research - Current Status
}

\author{
Michael Schmidt ${ }^{1}$, Sergei Rudenko¹, Klaus Börger² \\ 1 Deutsches Geodätisches Forschungsinstitut der Technischen Universität München (DGFI-TUM), Munich, Germany, \\ Contact: mg.schmidt@tum.de \\ 2 German Space Situational Awareness Centre (GSSAC), Uedem, Germany
}

\section{Space Weather}

- Space weather is a very up-to-date and interdisciplinary field of research.

- It describes physical processes in space mainly caused by the Sun's radiation of energy and plasma release.

- There are multiple manifestations of space weather, e.g., the variations of the Earth magnetic field, the polar lights as well as the variations of the ionosphere and thermosphere (due to coupled processes).

- The most extreme known space weather event happened at September 1, 1859 - the Carrington storm.

- Prominent recent events are the Halloween storm at October 28 - 30, 2003, the Bastille Day Event at July 14, 2000 or the St. Patrick's storm at March 17, 2015.

- The strength of these events, their impacts on modern society and the possibility of much stronger future events have brought several countries to recognize the necessity

- of studying these impacts scientifically,

- to establish space weather data centers and space weather services.

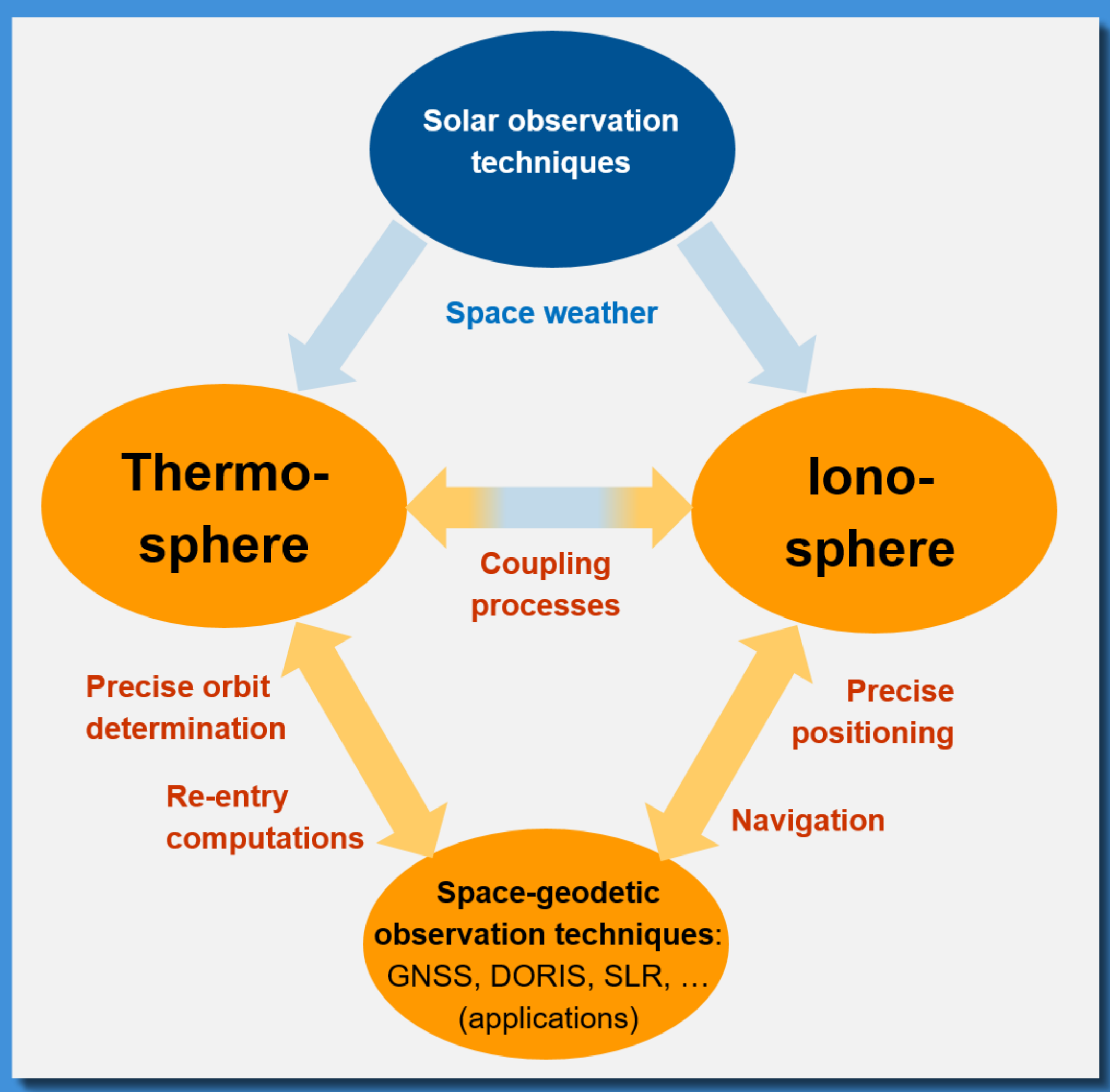

Figure 1: Structure of the FA-GSWR illustrated by a diamond. The orange parts can mainly be covered by geodesy (space-geodetic measurement techniques and evaluation methods); for the blue parts interdisciplinary studies and investigations have to be performed.

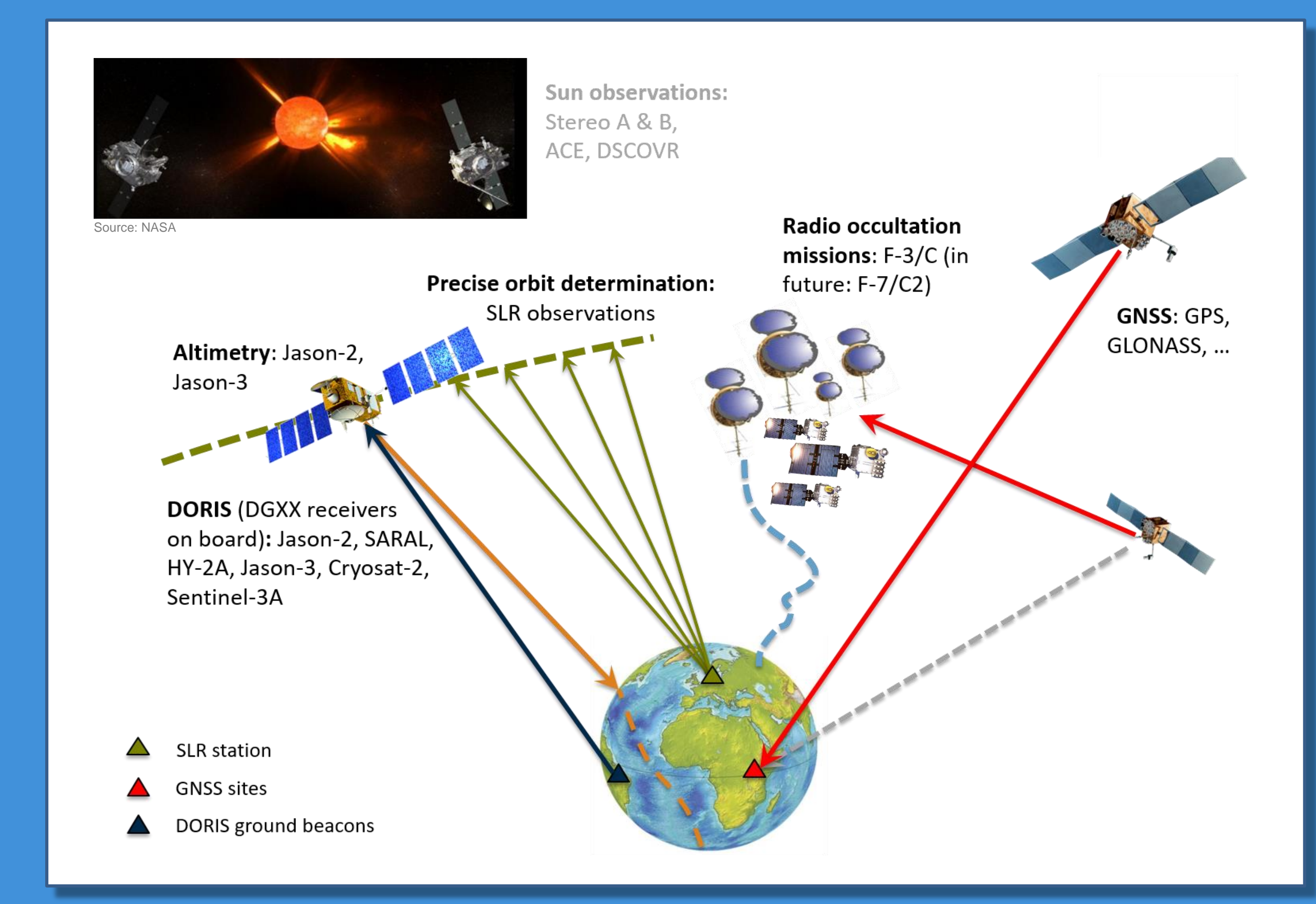

Figure 2: Space-geodetic observation techniques for monitoring and modelling the thermosphere-ionosphere system. Sun observations from solar missions will be used to forecast space weather events.

\section{Geodetic Monitoring}

- Figure 1 illustrates the structure of the Focus Area on Geodetic Space Weather Research (FA-GSWR) as a rhombus

- Satellite Geodesy deals for a long time with the ionosphere (PPP) and the thermosphere (POD)

- Thermospheric drag is the most important force acting on Low-Earth Orbiting (LEO) satellites and objects in the re-entry stage

- Figure 2 gives an overview about the space-geodetic observation techniques which provide valuable information about the coupled thermosphere-ionosphere (TIC) system

- The properties of the upper atmosphere have a strong impact on the execution of fundamental geodetic tasks such as positioning and gravity field modelling

- Geodesy has a long history and large experience in developing sophisticated analysis techniques and modelling approaches
Objectives of the FA-GSWR

The main objectives are:

- improvement of precise point positioning and navigation by developing high-precision and highresolution models of the electron density (PPP)

- improvement of precise orbit determination by developing highprecision and high-resolution thermospheric drag models (POD).

- Study of the coupled processes between thermosphere and ionosphere (TIC)
Joint Study Groups (JSG) and Joint Working Groups (JWG)

For the realization of the objectives one new GGOS JSG and three new GGOS JWGs will be installed:

- JSG 1: Improved understanding of the coupled processes (implemented within IAG ICCT and joint with GGOS); chair person: Andres Calabia Aibar

- JWG 1: Electron density modelling (joint with IAG Com. 4); chair person: Fabricio dos Santos Prol

- JWG 2: Thermosphere modelling including physics-based realisations of the coupled thermosphereionosphere processes (joint with IAG Com. 4); chair position: still vacant

- JWG 3: Improved understanding of space weather events and their monitoring by satellite missions (joint with IAG Com. 4); chair position still vacant

\section{Essential Geodetic Variables (EGV)}

- Since main geodetic tasks are depending on the properties of the upper atmosphere, the electron density and the neutral density are the most important EGVs from the view of the FA-GSWR. 